ANNALES

POLONICI MATHEMATICI

$88.2(2006)$

\title{
On absolutely monotone set-valued functions
}

\author{
by ANDrZej Smajdor (Kraków)
}

\begin{abstract}
We define absolutely monotone multifunctions and prove their analyticity on an interval $[0, b)$.
\end{abstract}

1. Let $f:[a, b) \rightarrow \mathbb{R}$. The $p$ th order difference $\Delta_{s}^{p} f(t)$ of $f$ is defined inductively as follows:

$$
\Delta_{s}^{0} f(t)=f(t), \quad \Delta_{s}^{p+1} f(t)=\Delta_{s}^{p} f(t+s)-\Delta_{s}^{p} f(t)
$$

for every nonnegative integer $p, t \in[a, b), s>0$ such that $t+(p+1) s<b$.

We say that the function $f$ is absolutely monotone in the interval $[a, b)$ if $\Delta_{s}^{p} f(t) \geq 0$ for $a \leq t \leq t+p s<b, p=0,1, \ldots$ The following Bernstein theorem is well known (see e.g. [3, Theorem 2.3.2]):

THEOREM. Every absolutely monotone function $f:[0, b) \rightarrow \mathbb{R}$ is analytic:

in $[0, b)$ with $a_{n} \geq 0, n=0,1, \ldots$

$$
f(t)=\sum_{n=0}^{\infty} a_{n} t^{n}
$$

2. In this paper we prove an analogue of S. Bernstein's theorem for absolutely monotone set-valued functions. Let $Y$ be a real normed space and let $\operatorname{cc}(Y)$ denote the family of all nonempty compact convex subsets of $Y$. A set $C \in \operatorname{cc}(Y)$ is the Hukuhara difference of $A \in \operatorname{cc}(Y)$ and $B \in \operatorname{cc}(Y)$ if

$$
A=B+C=\{b+c: b \in B, c \in C\}
$$

(see [2]). If the difference $C=A-B$ exists, then it is unique. This is a consequence of the following:

Lemma 1 (cf. [5]). Let $A, B$ and $C$ be subsets of a real topological vector space such that

$$
A+B \subset C+B .
$$

If $C$ is convex closed and $B$ is nonempty bounded, then $A \subset C$.

2000 Mathematics Subject Classification: 26A48, 26A51, 26E25.

Key words and phrases: absolutely monotone set-valued functions. 
Now, let $-\infty<a<b \leq \infty$ and let $H:[a, b) \rightarrow \operatorname{cc}(Y)$. We define the $p$ th differences $\Delta_{s}^{p} H(t)$ by the recurrence

$$
\Delta_{s}^{0} H(t)=H(t), \quad \Delta_{s}^{p+1} H(t)=\Delta_{s}^{p} H(t+s)-\Delta_{s}^{p} H(t)
$$

for every nonnegative integer $p, t \in[a, b), s>0$ such that $t+(p+1) s<b$.

A set-valued function is said to be absolutely monotone if all differences $\Delta_{s}^{p} H(t)$ exist and each contains zero.

Example. Let $A \in \operatorname{cc}(Y)$ be such that $0 \in A$. Suppose that $h$ : $[a, b) \rightarrow[0, \infty)$. Then $H(t)=h(t) A$ is an absolutely monotone set-valued function if and only if $h$ is an absolutely monotone real function.

We can observe the following:

Remark. Let $b$ and $\alpha$ be positive numbers, $H:[0, b) \rightarrow \operatorname{cc}(Y)$ and $G(t)=H(\alpha t)$ on $[0, b / \alpha)$. Then $G$ is absolutely monotone if and only if $H$ is absolutely monotone.

Let $G:[0,1] \rightarrow \operatorname{cc}(Y)$ be a given multifunction. The polynomial

$$
B_{n}(t)=\sum_{i=0}^{n}\left(\begin{array}{c}
n \\
i
\end{array}\right) t^{i}(1-t)^{n-i} G\left(\frac{i}{n}\right)
$$

is called the $n$th Bernstein polynomial of $G$.

Theorem 1. If $G:[0,1] \rightarrow \operatorname{cc}(Y)$ is continuous (with respect to the Hausdorff metric $d$ in $\operatorname{cc}(Y))$, then

$$
d\left(B_{n}(t), G(t)\right) \leq \frac{3}{2} \omega\left(\frac{1}{\sqrt{n}}\right)
$$

where

$$
\omega(\delta)=\sup \left\{d\left(G\left(t^{\prime \prime}\right), G\left(t^{\prime}\right)\right):\left|t^{\prime \prime}-t^{\prime}\right|<\delta\right\} .
$$

The proof of this theorem runs similarly to the proof of Bernstein's approximation theorem (cf. [3]).

Lemma 2. Let $G:[0,1] \rightarrow \operatorname{cc}(Y)$ be a multifunction. Then

$$
B_{n}(t)=\sum_{i=0}^{n}\left(\begin{array}{c}
n \\
i
\end{array}\right) t^{i} \Delta_{1 / n}^{i} G(0)
$$

for positive integers $n$ and $t \in[0,1]$.

Proof. Let " $\sim$ " denote the Rådström equivalence relation between pairs of members of $\operatorname{cc}(Y)$ defined by the formula

$$
(A, B) \sim(C, D) \Leftrightarrow A+D=B+C .
$$

For any pair $(A, B),[A, B]$ denotes its equivalence class. All equivalence classes form a linear space $\widetilde{Y}$ with addition defined by the rule

$$
[A, B]+[C, D]=[A+C, B+D]
$$


and scalar multiplication

$$
\lambda[A, B]= \begin{cases}{[\lambda A, \lambda B]} & \text { for } \lambda \geq 0 \\ \lambda[A, B]=[-\lambda B,-\lambda A] & \text { for } \lambda<0\end{cases}
$$

(cf. [5]).

Consider the function $g:[0,1] \rightarrow \widetilde{Y}$ defined as follows:

$$
g(t)=[G(t),\{0\}] .
$$

It can be proved by induction that

$$
\Delta_{s}^{p} g(t)=\left[\Delta_{s}^{p} G(t),\{0\}\right]
$$

and

$$
\Delta_{s}^{p} g(t)=\sum_{i=0}^{p}\left(\begin{array}{l}
p \\
i
\end{array}\right) t^{i}(-1)^{p-i} g(t+i s)
$$

for nonnegative integers $p, t \in[0,1)$ and $s>0$ such that $t+p s<1$.

Let $b_{n}$ be Bernstein's polynomials of $g$ :

$$
b_{n}(t)=\sum_{i=0}^{n}\left(\begin{array}{l}
n \\
i
\end{array}\right) t^{i}(1-t)^{n-i} g\left(\frac{i}{n}\right) .
$$

Then

$$
b_{n}(t)=\left[B_{n}(t),\{0\}\right] .
$$

Using Newton's binomial formula, replacing $j$ by $j-i$ in the second sum below, changing the order of summation, and then making use of the identity $\left(\begin{array}{c}n \\ i\end{array}\right)\left(\begin{array}{c}n-i \\ j-i\end{array}\right)=\left(\begin{array}{c}n \\ j\end{array}\right)\left(\begin{array}{l}j \\ i\end{array}\right)$ and equality $(3)$ we obtain

$$
\begin{aligned}
b_{n}(t) & =\sum_{i=0}^{n} \sum_{j=0}^{n-i}\left(\begin{array}{c}
n \\
i
\end{array}\right)\left(\begin{array}{c}
n-i \\
j
\end{array}\right)(-1)^{j} t^{i+j} g\left(\frac{i}{n}\right) \\
& =\sum_{i=0}^{n} \sum_{j=i}^{n}\left(\begin{array}{c}
n \\
i
\end{array}\right)\left(\begin{array}{c}
n-i \\
j-i
\end{array}\right)(-1)^{j-i} t^{j} g\left(\frac{i}{n}\right) \\
& =\sum_{j=0}^{n} \sum_{i=0}^{j}\left(\begin{array}{c}
n \\
j
\end{array}\right)\left(\begin{array}{l}
j \\
i
\end{array}\right)(-1)^{j-i} t^{j} g\left(\frac{i}{n}\right)=\sum_{j=0}^{n}\left(\begin{array}{c}
n \\
j
\end{array}\right) t^{j} \Delta_{1 / n}^{j} g(0) .
\end{aligned}
$$

According to (2) we have

$$
\begin{aligned}
{\left[\sum_{i=0}^{n}\left(\begin{array}{c}
n \\
i
\end{array}\right) t^{i} \Delta_{1 / n}^{i} G(0),\{0\}\right] } & =\sum_{i=0}^{n}\left(\begin{array}{c}
n \\
i
\end{array}\right) t^{i}\left[\Delta_{1 / n}^{i} G(0),\{0\}\right] \\
& =\sum_{i=0}^{n}\left(\begin{array}{c}
n \\
i
\end{array}\right) t^{i} \Delta_{1 / n}^{i} g(0)=b_{n}(t)=\left[B_{n}(t),\{0\}\right] .
\end{aligned}
$$


Thus

$$
\left(\sum_{i=0}^{n}\left(\begin{array}{c}
n \\
i
\end{array}\right) t^{i} \Delta_{1 / n}^{i} G(0),\{0\}\right) \sim\left(B_{n}(t),\{0\}\right)
$$

and (1) holds.

Lemma 3. Let $0<c \leq b$ and $H:[0, b) \rightarrow \operatorname{cc}(Y)$. If $A_{i}, B_{i} \in \operatorname{cc}(Y)$, $i=0,1, \ldots$, are such that

$$
\begin{aligned}
& H(t)=\sum_{n=0}^{\infty} t^{n} A_{n} \quad \text { for } t \in[0, b), \\
& H(t)=\sum_{n=0}^{\infty} t^{n} B_{n} \quad \text { for } t \in[0, c),
\end{aligned}
$$

then $A_{i}=B_{i}$ for $i=0,1, \ldots$

Proof. We see that $A_{0}=H(0)=B_{0}$. Suppose that

$$
A_{0}=B_{0}, \ldots, A_{k}=B_{k} .
$$

Then

$$
A_{k+1}=\lim _{t \rightarrow 0+} \frac{H(t)-\sum_{i=0}^{k} t^{i} A_{i}}{t^{k+1}}=\lim _{t \rightarrow 0+} \frac{H(t)-\sum_{i=0}^{k} t^{i} B_{i}}{t^{k+1}}=B_{k+1} .
$$

THEOREM 2. A set-valued function $H:[0, b) \rightarrow \operatorname{cc}(Y)$ is absolutely monotone if and only if there exist sets $A_{i} \in \operatorname{cc}(Y), i=0,1, \ldots$, containing zero such that

$$
H(t)=\sum_{n=0}^{\infty} t^{n} A_{n} \quad \text { for } t \in[0, b)
$$

Proof. 1. Suppose that $H:[0, b) \rightarrow \operatorname{cc}(Y)$ is of the form (4) and that $0 \in A_{n} \in \operatorname{cc}(Y)$. We see that

$$
H(t+s)=\sum_{n=0}^{\infty}(t+s)^{n} A_{n}=H(t)+\sum_{n=0}^{\infty}\left((t+s)^{n}-t^{n}\right) A_{n},
$$

therefore

$$
\Delta_{s}^{1} H(t)=\sum_{n=0}^{\infty} \Delta_{s}^{1} t^{n} A_{n}
$$

By induction it may be shown that

$$
\Delta_{s}^{p} H(t)=\sum_{n=0}^{\infty} \Delta_{s}^{p} t^{n} A_{n}
$$

Thus all differences $\Delta_{s}^{p} H(t)$ exist. As they contain zero, $H$ is an absolutely monotone multifunction. 
2. Now, suppose that $H:[0, b) \rightarrow \operatorname{cc}(Y)$ is an absolutely monotone multifunction. The differences $\Delta_{s}^{1} H(t)$ and $\Delta_{s}^{2} H(t)$ exist and contain zero, therefore

$$
H(t) \subset H(t)+\Delta_{s}^{1} H(t)=H(t+s)
$$

and

$$
\begin{aligned}
2 H(t+s) & \subset 2 H(t+s)+\Delta_{s}^{2} H(t)=H(t+s)+H(t)+\Delta_{s}^{1} H(t)+\Delta_{s}^{2} H(t) \\
& =H(t+s)+H(t)+\Delta_{s}^{1} H(t+s)=H(t)+H(t+2 s) .
\end{aligned}
$$

Thus $H$ is increasing and midconcave in $[0, b)$.

Fix a number $c \in(0, b)$. The function $H$, being midconcave and bounded on $[0, c]$, is continuous, according to Theorem 4.4 in [4]. Define $G(t)=H(c t)$ for $t \in[0,1]$. Then $G$ is continuous and by Theorem 1 it is the uniform limit of the sequence of its Bernstein polynomials $B_{n}(t)$. By Lemma 2 we have

$$
B_{n}(t)=\sum_{i=0}^{n}\left(\begin{array}{c}
n \\
i
\end{array}\right) t^{i} \Delta_{1 / n}^{i} G(0)=\sum_{i=0}^{n} t^{i} A_{i}^{n},
$$

where $A_{i}^{n}=\left(\begin{array}{c}n \\ i\end{array}\right) \Delta_{1 / n}^{i} G(0)$. We note that $0 \in A_{0}^{n}$ and

$$
A_{0}^{n} \subset B_{n}(1)=G(1) .
$$

Since $G(1)$ is compact, the family of all closed subsets of $G(1)$ is compact (see $[1$, p. 41$])$. By $(5)$ there exists a strictly increasing sequence $\left(n_{k}^{0}\right)$ and $A_{0}(c) \in \operatorname{cc}(Y)$ such that

$$
A_{0}^{n_{k}^{0}} \rightarrow A_{0}(c)
$$

Similarly, since

$$
A_{1}^{n_{k}^{0}} \subset B_{n_{k}^{0}}(1)=G(1),
$$

there exists a strictly increasing subsequence $\left(n_{k}^{1}\right)$ of $\left(n_{k}^{0}\right)$ and $A_{1} \in \operatorname{cc}(Y)$ such that

$$
A_{0}^{n_{k}^{1}} \rightarrow A_{1}(c)
$$

and so on. Applying the diagonalization procedure to the sequences $\left(A_{0}^{n_{k}^{0}}\right)$, $\left(A_{0}^{n_{k}^{1}}\right), \ldots$ we obtain a strictly increasing sequence $\left(n_{k}\right)$ such that

$$
A_{0}^{n_{k}} \rightarrow A_{0}(c), A_{1}^{n_{k}} \rightarrow A_{1}(c), \ldots
$$

Fix $t \in[0,1), \varepsilon>0$ and define

$$
S_{n}(t)=\sum_{i=0}^{n} t^{i} A_{i}(c) \quad \text { for } n=0,1, \ldots
$$

Choose a positive integer $k$ so large that $2\|G(1)\| t^{k}(1-t)^{-1}<\varepsilon / 3$, where $\|G(1)\|=\sup \{\|y\|: y \in G(1)\}$, and then choose $L$ large enough to get $d\left(B_{n_{l}}(t), G(t)\right)<\varepsilon / 3$ and $\sum_{j=0}^{k-1} d\left(A_{j}^{n_{l}}, A_{j}\right)<\varepsilon / 3$ for $l \geq L$. Then 


$$
\begin{aligned}
d\left(S_{n_{l}}(t), G(t)\right) & \leq d\left(S_{n_{l}}(t), B_{n_{l}}(t)\right)+d\left(B_{n_{l}}(t), G(t)\right) \\
& \leq 2 \varepsilon / 3+\sum_{i=k+1}^{n_{l}} t^{i} d\left(A_{i}^{n_{l}}, A_{i}\right) \leq(2 / 3) \varepsilon+2\|G(1)\| \frac{t^{k+1}}{1-t}<\varepsilon
\end{aligned}
$$

Thus

$$
\lim _{l \rightarrow \infty} S_{n_{l}}(t)=G(t)
$$

and according to Theorem II-2 in [1],

$$
G(t)=\bigcup_{l=1}^{\infty} S_{n_{l}}(t) .
$$

Using the monotonicity of the sequence $\left(S_{n}(t)\right)$ we get

$$
S_{l}(t) \subset S_{n_{l}}(t) \subset G(t) \quad \text { for } l=0,1, \ldots
$$

Therefore the sequence $d\left(G(t), S_{l}(t)\right)$ is decreasing. By (6),

Consequently,

$$
\lim _{l \rightarrow \infty} d\left(G(t), S_{l}(t)\right)=\lim _{l \rightarrow \infty} d\left(G(t), S_{n_{l}}(t)\right)=0 .
$$

$$
G(t)=\lim _{n \rightarrow \infty} S_{n}(t)=\sum_{i=0}^{\infty} t^{i} A_{i} \quad \text { for } t \in[0,1) .
$$

The definition of $G$ leads to

$$
H(t)=G(t / c)=\sum_{i=0}^{\infty} t^{i} c^{-i} A_{i} \quad \text { for } t \in[0, c) .
$$

Now (4) follows from Lemma 3.

\section{References}

[1] C. Castaing and M. Valadier, Convex Analysis and Measurable Multifunctions, Lecture Notes in Math. 580, Springer, Berlin, 1977.

[2] M. Hukuhara, Intégration des applications mesurables dont la valeur est un compact convexe, Funcial. Ekvac. 10 (1967), 205-223;

[3] S. Łojasiewicz, An Introduction to the Theory of Real Functions, Wiley, 1988.

[4] K. Nikodem, K-convex and K-concave set-valued functions, Zeszyty Nauk. Politech. Łódz. Mat. 559, Rozprawy Naukowe 114, 1989.

[5] H. Rådström, An embedding theorem for spaces of convex sets, Proc. Amer. Math. Soc. 3 (1952), 165-169.

Pedagogical University

Podchorążych 2

30-084 Kraków, Poland

E-mail: asmajdor@ap.krakow.pl 